\title{
Axion-like Particles from Nearby Type la Supernovae
}

\author{
Kanji Mori ${ }^{1, *}$ \\ ${ }^{1}$ Research Institute of Stellar Explosive Phenomena, Fukuoka University, 8-19-1 Nanakuma, Jonan-ku, \\ Fukuoka-shi, Fukuoka 814-0180, Japan
}

\begin{abstract}
Axion-like particles (ALPs) are a class of hypothetical pseudo-scalar particles and can be created in hot astrophysical plasma through the interaction between photons. I calculate the production of ALPs in type Ia supernovae. It is found that many ALPs lighter than a few MeV can be produced in type Ia supernovae. Once produced, heavy ALPs decay into photons during propagation in the interstellar space. I calculate the flux of the decay photons and find that it may be detected by future $\mathrm{MeV} \gamma$-ray telescopes if a type Ia supernova explodes near the Solar System.
\end{abstract}

\section{Introduction}

Axion-like particles (ALPs) are hypothetical pseudo Nambu-Goldstone bosons which can couple with photons. The ALP-photon interaction is described by the Lagrangian

$$
\mathcal{L}_{a \gamma \gamma}=-\frac{1}{4} g_{a \gamma} a \tilde{F}^{\mu v} F_{\mu \nu}
$$

where $g_{a \gamma}$ is the coupling constant, $a$ is the ALP field, $F_{\mu \nu}$ is the electromagnetic tensor, and $\tilde{F}^{\mu v}$ is its dual. Axions were first introduced to solve the strong CP problem in quantum chromodynamics (QCD) [1,2]. Since then, the concept of QCD axions has been generalized as ALPs to describe similar exotic particles [e.g. 3].

Astrophysical objects such as stars and supernovae have been used to constrain heavy ALPs. Since ALPs can induce additional energy losses from stars and affect the stellar lifetime, observations of globular clusters have provided a constraint on ALPs [4]. Also, the additional energy losses may prevent neutrino bursts in core-collapse supernovae [5]. ALP parameters can be constrained by comparing the model calculation and neutrino observations of SN 1987A. Once produced in an astronomical object, heavy ALPs decay into photons during propagation in the interstellar space. The non-detection of decay photons from SN 1987A provided a stringent constraint [6].

Supernovae are classified into two classes: core-collapse and thermonuclear supernovae. Core-collapse supernovae are the death of massive stars. On the other hand, thermonuclear supernovae are thermonuclear explosions of white dwarfs and called type Ia supernovae ( $\mathrm{SNe}$ Ia). Previous works on ALPs from supernovae have focused on core-collapse supernovae. In this study, we calculate the production of ALPs in a nearby SN Ia and the flux of decay photons [7].

\footnotetext{
*e-mail: kanji.mori@fukuoka-u.ac.jp
} 


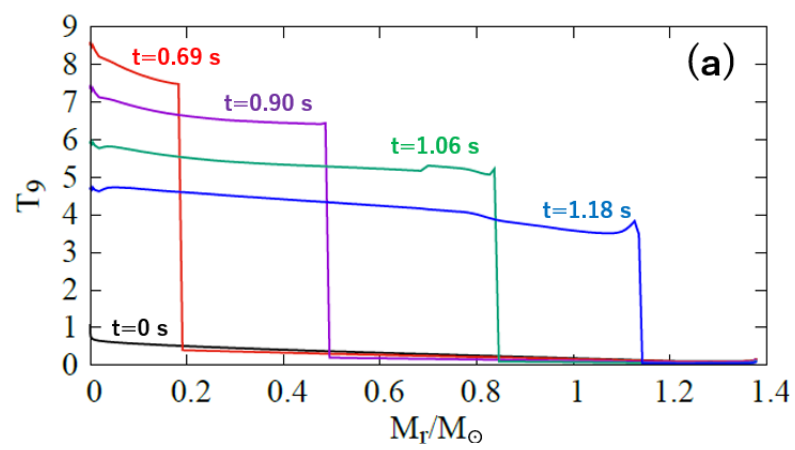

Figure 1. The temperature profile in the W7 model [7].

\section{Method}

We adopt the W7 model as a SN Ia model. W7 is a one-dimensional deflagration model with a near Chandrasekhar carbon-oxygen white dwarf [8]. Fig. 1 shows the temperature $T_{9}$ in unit of $10^{9} \mathrm{~K}$ as a function of the mass coordinate $M_{r}$. The ignition starts at the center and the deflagration propagates toward the surface.

We consider the Primakoff process and the photon coalescence as ALP production processes. The rate of the Primakoff process is calculated as [7, 9]

$$
\begin{array}{r}
\Gamma_{\gamma \rightarrow a}=g_{a \gamma}^{2} \frac{T \kappa^{2}}{32 \pi} \frac{p}{E}\left(\frac{\left((k+p)^{2}+\kappa^{2}\right)\left((k-p)^{2}+\kappa^{2}\right)}{4 k p \kappa^{2}} \ln \left(\frac{(k+p)^{2}+\kappa^{2}}{(k-p)^{2}+\kappa^{2}}\right)\right. \\
\left.-\frac{\left(k^{2}-p^{2}\right)^{2}}{4 k p \kappa^{2}} \ln \left(\frac{(k+p)^{2}}{(k-p)^{2}}\right)-1\right),
\end{array}
$$

where $T$ is the temperature, $\kappa$ is the inverse Debye-Hückel length, $p$ and $E$ are the ALP momentum and energy, and $k$ is the momentum of plasmons. The rate for the photon coalescence is calculated as

$$
\frac{d^{2} n_{a}}{d t d E}=g_{a \gamma}^{2} \frac{m_{a}^{4}}{128 \pi^{3}} p\left(1-\frac{4 \omega_{\mathrm{pl}}^{2}}{m_{a}^{2}}\right)^{\frac{3}{2}} e^{-\frac{E}{T}},
$$

where $m_{a}$ is the ALP mass and $\omega_{\mathrm{pl}}$ is the plasma frequency. The contribution of the photon coalescence to the total ALP production becomes significant when ALPs are heavier than $\sim 1$ $\mathrm{MeV}$.

Heavy ALPs decay radiatively during the propagation. The mean free path is calculated as $\lambda=\beta \gamma / \Gamma_{a \rightarrow \gamma \gamma}[6,7]$, where $\beta$ is the ALP velocity, $\gamma$ is the Lorentz factor, and $\Gamma_{a \rightarrow \gamma \gamma}=$ $g_{a \gamma}^{2} m_{a}^{3} / 64 \pi$.

\section{Results}

Fig. 2 shows the ALP luminosity defined as

$$
L_{a}=4 \pi \int Q_{a} r^{2} d r
$$

where $r$ is the radius and $Q_{a}$ is the ALP emissivity per unit volume and time. In the plot, the ALP-photon coupling is fixed to $g_{10}=1$. It is seen that $L_{a}$ reaches its peak at $\sim 1 \mathrm{~s}$ 


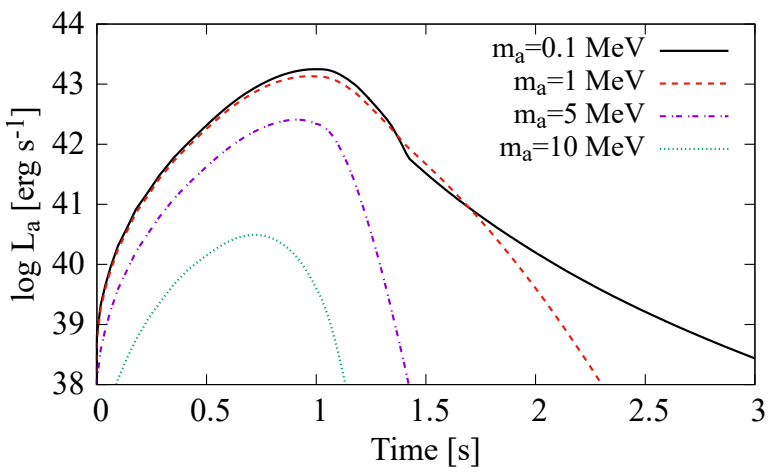

Figure 2. The ALP luminosity as a function of time after the ignition [7]. The ALP-photon coupling is fixed to $g_{10}=1$.
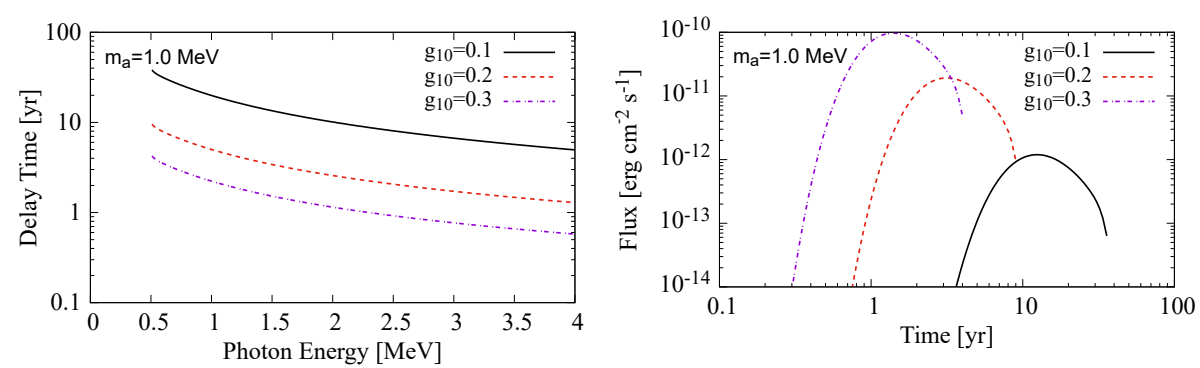

Figure 3. The delay time (left) and the energy flux (right) of decay photons from an SN Ia located 1 $\mathrm{kpc}$ away from the Solar System [7]. The ALP mass is fixed to $m_{a}=1 \mathrm{MeV}$.

after ignition. When ALPs are heavier than $\sim 5 \mathrm{MeV}, L_{a}$ is suppressed. This is because the temperature in $\mathrm{SNe}$ Ia is lower than $1 \mathrm{MeV}$ and the plasma is not energetic enough to produce such heavy particles.

In this study, we assume that an SN Ia is located $1 \mathrm{kpc}$ away from the Solar System. Since ALPs are slower than photons, decay photons would be observed later than the supernova event. The delay time is calculated as $t=L_{1} / \beta+L_{2}-d_{\mathrm{SN}}$, where $L_{1} \sim \lambda$ is the distance which ALPs travel, $L_{2}$ is the distance which decay photons travel, and $d_{\mathrm{SN}}$ is the distance between the SN Ia and the Solar System. Fig. 3 shows the delay time as a function of the energy of decay photons. One can see that more energetic photons arrive on Earth earlier. In this ALP parameter range, typical values of the delay time are $1-10$ years.

Fig. 3 also shows the energy flux $F(t)=E_{\gamma}(t) d n_{\gamma}(t) / d t$ as a function of time after the SN explosion. Here $E_{\gamma}(t)$ is the energy of decay photons and $n_{\gamma}(t)$ is the number flux of decay photons that arrive on Earth. The number flux is given as $[7,10]$

$$
\frac{d n_{\gamma}}{d t}=\frac{2}{4 \pi d_{\mathrm{SN}}^{2}} \frac{d n_{a}}{d E_{a}}\left(\frac{d t}{d E_{a}}\right)^{-1} e^{-\frac{R_{\mathrm{SN}}}{\lambda\left(E_{a}\right)}}\left(1-e^{-\frac{d_{\mathrm{SN}}}{\lambda\left(E_{a}\right)}}\right),
$$

where the factor 2 represents two photons that are produced from an ALP, $d_{\mathrm{SN}}=1 \mathrm{kpc}$ is the distance to the event, $E_{a}$ is the ALP energy, $n_{a}$ is the number of ALPs, and $R_{\mathrm{SN}}$ is the radius of the star. The formula takes into account the effect of the ALP decay in the star and in the interstellar space. From Fig. 3, we see that the flux increases as a function of $g_{10}$. 
When $g_{10} \gtrsim 0.2$, the flux reaches $\sim 10^{-12} \mathrm{erg} \mathrm{cm}^{-2} \mathrm{~s}^{-1}$. This flux may be observed by nextgeneration $\mathrm{MeV} \gamma$-ray telescopes such as All-sky Medium Energy Gamma-ray Observatory [AMEGO; 11] and e-ASTROGAM [12]. (Non-)detection of decay photons would provide independent information on the ALP parameters.

In this study, we adopted a near-Chandrasekhar model of SNe Ia. However, the nature of SN Ia progenitors is still under debate. Recently, sub-Chandrasekhar models are being investigated as an alternative scenario [e.g. 13, 14]. Since the density in sub-Chandrasekhar white dwarfs is much lower, the ALP production there can be significantly different from the results for Chandrasekhar models. It is desirable to perform systematic studies for various models to understand the model dependence in future studies.

This work is supported by Research Institute of Stellar Explosive Phenomena at Fukuoka University and JSPS KAKENHI grant number JP21K20369.

\section{References}

[1] S. Weinberg, Phys. Rev. Lett. 40, 223 (1978)

[2] F. Wilczek, Phys. Rev. Lett. 40, 279 (1978)

[3] K. Choi, S.H. Im, C.S. Shin, arXiv e-prints arXiv:2012.05029 (2020), 2012.05029

[4] P. Carenza, O. Straniero, B. Döbrich, M. Giannotti, G. Lucente, A. Mirizzi, Physics Letters B 809, 135709 (2020), 2004.08399

[5] G. Lucente, P. Carenza, T. Fischer, M. Giannotti, A. Mirizzi, J. Cosmol. Astropart. Phys. 2020, 008 (2020), 2008.04918

[6] J. Jaeckel, P.C. Malta, J. Redondo, Phys. Rev. D 98, 055032 (2018), 1702 . 02964

[7] K. Mori, Publ. Astron. Soc. Japan 73, 1382-1388 (2021), 2107.09097

[8] K. Nomoto, F.K. Thielemann, K. Yokoi, Astrophys. J. 286, 644 (1984)

[9] L. di Lella, A. Pilaftsis, G. Raffelt, K. Zioutas, Phys. Rev. D 62, 125011 (2000), hep-ph/0006327

[10] A. Caputo, P. Carenza, G. Lucente, E. Vitagliano, M. Giannotti, K. Kotake, T. Kuroda, A. Mirizzi, Phys. Rev. Lett. 127, 181102 (2021), 2104.05727

[11] J. McEnery, A. van der Horst, A. Dominguez, A. Moiseev, A. Marcowith, A. Harding, A. Lien, A. Giuliani, A. Inglis, S. Ansoldi et al., All-sky Medium Energy Gamma-ray Observatory: Exploring the Extreme Multimessenger Universe, in Bulletin of the American Astronomical Society (2019), Vol. 51, p. 245, 1907.07558

[12] A. de Angelis, V. Tatischeff, I.A. Grenier, J. McEnery, M. Mallamaci, M. Tavani, U. Oberlack, L. Hanlon, R. Walter, A. Argan et al., Journal of High Energy Astrophysics 19, 1 (2018), 1711.01265

[13] K.J. Shen, D. Kasen, B.J. Miles, D.M. Townsley, Astrophys. J. 854, 52 (2018), 1706.01898

[14] S.C. Leung, K. Nomoto, Astrophys. J. 888, 80 (2020), 1901.10007 